\title{
Impact of Heat Shock Proteins in Hepatocellular Carcinoma
}

\author{
Mihir Y Parmar ${ }^{*}$, Sachin Kumar Sharma ${ }^{2}$ and Dinesh Pore $^{1}$ \\ ${ }^{1}$ Bharat Institute of Technology, Jawaharlal Nehru Technological University, India \\ ${ }^{2}$ Parul Institute of Pharmacy and Research, Parul University, India
}

Submission: November 20, 2018; Published: December 05, 2018

*Corresponding author: Mihir Y Parmar, Bharat Institute of Technology, Mangalpally, Ibrahimpatnam, Jawaharlal Nehru Technological University, Hyderabad, Telangana, India

\begin{abstract}
Heat shock proteins are highly conserved proteins, expressed at low levels under normal conditions. Heat shock proteins significantly induced in response to cellular stresses and lead to heat shock response, which could help cancer cells to adapt to stress conditions. As molecular chaperones, Heat shock proteins play critical roles in protein homeostasis, apoptosis, invasion and cellular signaling transduction. A heat shock protein over expression is widely reported in many human cancers due to constant stress condition in tumor microenvironment. Heat shock proteins often associated with poor prognosis in many types of human cancers. Up regulation of Heat shock proteins may serve as diagnostic and prognostic markers in hepatocellular carcinoma. Targeting Heat shock proteins with specific inhibitor alone or in combination with chemotherapy regimens holds promise for the improvement of outcomes for hepatocellular carcinoma patients. In addition, our study suggests progression and challenges in targeting these Heat shock proteins as novel therapeutic strategies in hepatocellular carcinoma.
\end{abstract}

Keywords: Heat shock proteins; Hepatocellular carcinoma; Heat shock response

Abbreviations: HSPs: Heat Shock Proteins; HCC: Hepatocellular Carcinoma; HSF1: Heat Shock Factor

\section{Introduction}

Heat shock proteins (HSPs) are ubiquitous proteins found in the cells of all studied organisms, which are expressed at low levels under normal conditions. Many types of stress, including heat, induce expression of a family of genes known as the heat shock protein genes. Heat shock proteins originally were discovered when it was observed that heat shock produced chromosomal puffs in the salivary glands of fruit flies (Drosophilia) [1-2]. The DNA sequence that makes up this family of genes is highly conserved across species. This family of genes originally was named because of their expression after exposure to heat. However, the genes are now known to be induced by a wide variety of environmental or metabolic stresses that include the following: anoxia (hypoxia), ischemia, heavy metal ions, ethanol, nicotine, surgical stress, viral agents, genotoxic agents, nutrient starvation and overexpression of oncoproteins [3].

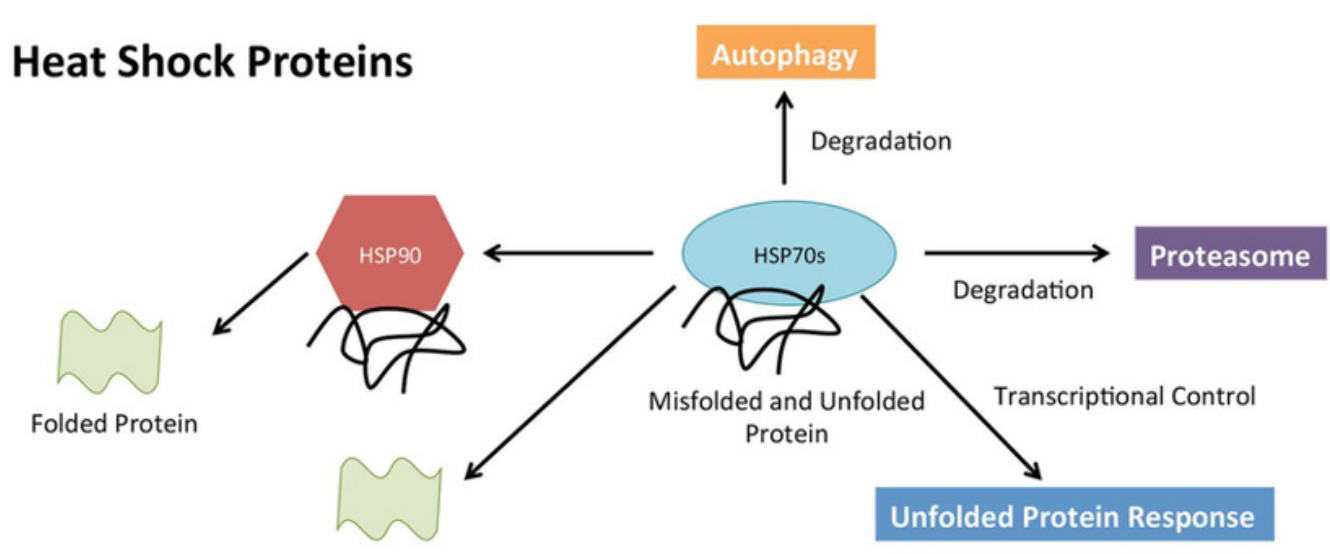

Folded Protein

Figure 1: Heat shock proteins (HSPs) role in proteostasis. Both HSP70 and HSP90 are able to orchestrate and facilitate, with the assistance of other molecular pathways, the folding and degradation of unfolded/misfolded proteins within the cell. 


\section{International Journal of Cell Science \& Molecular Biology}

Thus, the term "heat shock protein" is a misnomer because many agents other than heat induce the expression of the heat shock protein gene. Consequently, "stress protein" is the preferred term. Stress proteins are critically important because they appear to be necessary in the critical step of three-dimensional folding of some newly formed proteins within the cell. In fact, they ensure that newly formed polypeptides proceed correctly through folding and unfolding to eventually achieve a functional shape (Figure 1). Stress proteins also assist in the repair of denatured proteins or promote their degradation after stress or injury. They have been referred to as "molecular chaperones" because of this function $[1,4]$.

Upregulation of HSPs is a critical part of heat shock response, which could help cells to cope with the stress condition. Many members of HSPs perform their functions as molecular chaperons by stabilizing proteins to ensure their correct folding, inhibiting stress-induced protein aggregation or regulating cellular signalling and transcriptional networks. The increased expression of HSPs under stress condition is often transcriptionally regulated by heat shock factor 1 (HSF1). In response to stress conditions, HSF1 is phosphorylated and forms homotrimers, then binds to heat shock elements (HSEs) located upstream of HSPs genes and activates the transcription of heat shock genes [5-6]. Stress proteins belong to a multigene family and often named according to their molecular weight from 8 to $150 \mathrm{kd}$. In this review, we will discuss three major members of the HSP family, which are closely related to human malignancies, namely HSP27, HSP70 and HSP90. It is thought that stress proteins are produced in response to nonlethal stress to protect organisms from subsequent severe stress that would otherwise be lethal. In the case of exposure to heat, this phenomenon has been called "thermotolerance" and has launched many experiments in which an association has been found between the heat shock response and protection against other stresses, such as hypoxia or ischemia. The addition of one type of stress may provide protection against other types of insults, which results in cross-tolerance. As examples, stress protein induction by hyperthermia may provide protection during a subsequent arterial injury or exposure to a heavy metal may provide subsequent protection against heat or ischemic injury. This thermotolerance treatment strategy has proved successful in experimental models of cardiac ischemia, arterial injury, endotoxic shock, renal and hepatic ischemia, ethanol-induced gastric ulcerations, and skeletal muscle ischemia-reperfusion [7]. HSP27 overexpression is reported in a wide range of human cancers. Report shows that HSP27 performs crucial roles in cell cycle modulation, apoptosis inhibition, cytoskeleton organization, regulation of translation initiation, DNA repair, RNA splicing and degradation of oxidized proteins via the ubiquitin-proteasome pathway.

Stress proteins play a critical role in the maintenance of normal cellular homeostasis. These proteins almost certainly have a pivotal role in cell cycle progression and cell death (apoptosis) and are involved in many disease processes, including cardiovascular disease. Currently, the manipulation of stress proteins remains cumbersome because hyperthermia and pharmacologic manipulations are relatively nonspecific. Eventually, as we gain more insight into the exact role and function of these fascinating molecules, the clinical manipulation of the stress proteins will almost certainly prove beneficial [8]. Liver cancer is one of the most frequent malignancies worldwide [9]. An estimated 782,500 new liver cancer cases and 745,500 deaths occurred worldwide during 2012 [10]. About half of the new cases and related deaths of liver cancer occurred in China. Hepatocellular carcinoma (HCC) accounts for more than $80 \%$ of primary liver cancers. Although treatment techniques for HCC have experienced great progress, prognosis is still poor for HCC patients. Only 30-40\% of HCC patients are suitable for curative treatment at the time of diagnosis. Long-term survival following radical surgical resection remains unsatisfactory because of high rates of recurrence and metastasis [11]. A better understanding of molecular mechanisms involved in tumorigenesis and metastasis of HCC will provide novel and potential therapeutic implications in HCC treatment. HSPs have become attractive therapeutic targets in HCC. Novel therapeutic strategies that target HSPs alone or combined with other anticancer agents are widely investigated [12].

\section{Conclusion and Future Directions}

As stress induction of HSPs plays a crucial role in tumorigenesis, metastasis and therapeutic resistance, the clinical efficacies of HSPs as biomarkers for the diagnosis and prognosis of HCC patients warrant further testing in clinical settings. Targeting HSPs with their specific inhibitors holds attractive therapeutic promise for the improvement of outcomes for HCC patients. Despite promising preclinical data, there are still some challenges to target HSPs in HCC. First, little is known about the therapeutic responses of HSPs inhibitors in HCC patients. Therefore, more clinical trials about the antitumor activity of HSPs inhibitors in HCC patients are urgently required. Second, the future of HSPs inhibitors in the treatment of cancer individuals may lie in combining them with cytotoxic chemotherapy or other targeted therapies. Although some preliminary studies have shown enhanced efficacy of HSPs inhibition combined with targeted drugs such as rapamycin and sorafenib, the best strategy for combination treatment still needs to be validated in further studies. Third, we can focus our attention on HSPs inhibitors directly. Intrinsic or acquired resistance often limits the efficiencies of HSPs inhibitors CRISPR/Cas9- or shRNAbased loss-of-function genetic screens can help us to identify mechanisms of the resistance and find potential combined drug targets whose inactivation is effective to improve efficiencies of HSPs inhibitors.123-125 Fourth, HSPs constitute 1-2\% of total proteins in most celltypes.126 HSPs are essential for both cancer and normal cells, and play important roles in a wide range of cellular processes such as maintaining protein homeostasis, intracellular trafficking, signal transduction and regulating innate immune responses. HSPs targeting may have unacceptable deleterious effects on non-malignant cells and normal organs in clinical trials. As a consequence, novel HSPs inhibitors with high selectivity and potency for tumor cells are eagerly to be developed. Finally, molecular mechanisms of HSPs in cytoprotective effects 
and tumor metastasis are still not fully understood. Answers to key issues of these basic mechanisms will significantly accelerate the applications of HSPs inhibitors in HCC treatment.

\section{References}

1. Feder ME, Hofmann GE (1999) Heat-shock proteins, molecular chaperones, and the stress response: evolutionary and ecological physiology. Annu Rev Physiol 61: 243-282.

2. Roberts RJ, Agius C, Saliba C, Bossier P, Sung YY (2010) Heat shock proteins (chaperones) in fish and shellfish and their potential role in relation to fish health: a review, J Fish Dis 33(10): 789-801.

3. David W, Steven P, William D, Birmingham (1999) Heat shock proteins: A review of the molecular chaperones. Journal of vascular surgery 29(4): 748-751.

4. Vaishali K, Melanie MB, Melania M, Serena C, Harm HK (2014) Barcoding heat shock proteins to human diseases: looking beyond the heat shock response. The Company of Biologists Ltd. Dis Model Mech 7: 421-434.

5. Dai C, Whitesell L, Rogers AB (2007) Heat shock factor 1 is a powerful multifaceted modifier of carcinogenesis. Cell 130(6): 1005-1018.

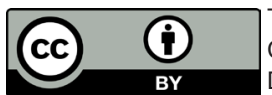

is work is licensed under Creative Commons Attribution 4.0 License

DOI: 10.19080/IJCSMB.2018.05.555663
6. Dudeja V, Vickers SM, Saluja AK (2009) The role of heat shock proteins in gastrointestinal diseases. Gut 58(7): 1000-1009.

7. Gopal KP, Arabinda M, Mrutyunjay S, Anil PS, Bimal PM, et al. (2014) Investigating hsp Gene Expression in Liver of Channa striatus under Heat Stress for Understanding the Upper Thermal Acclimation. BioMed Research International: 1-11.

8. Wen JLu, Nikki PL, Sarwat F, John ML (2009) Heat Shock Proteins in Cancer: Signalling Pathways, Tumor Markers and Molecular Targets in Liver Malignanc, Protein Pept Lett 16 (5): 508-516.

9. Jemal A, Bray F, Center MM (2011) Global cancer statistics. CA Cancer J Clin 61: 69-90

10. Forner A, Llovet JM, Bruix J (2012) Hepatocellular carcinoma. The Lancet 379(9822):1245-1255.

11. Portolani N, Coniglio A, Ghidoni S (2006) Early and late recurrence after liver resection for hepatocellular carcinoma: prognostic and therapeutic implications. Ann Surg 243: 229-235.

12. Wang C, Jin G, Jin H (2015) Clusterin facilitates metastasis by EIF3I/ Akt/MMP13 signaling in hepatocellular carcinoma. Oncotarget 6(5): 2903-2916.

\section{Your next submission with Juniper Publishers} will reach you the below assets

- Quality Editorial service

- Swift Peer Review

- Reprints availability

- E-prints Service

- Manuscript Podcast for convenient understanding

- Global attainment for your research

- Manuscript accessibility in different formats

( Pdf, E-pub, Full Text, Audio)

- Unceasing customer service

Track the below URL for one-step submission https://juniperpublishers.com/online-submission.php 